\title{
Emotional intelligence as a structure-forming component of the future teacher's psychological culture
}

\author{
O.A. Rudakova ${ }^{*}$, and E.Yu. Ponomareva ${ }^{2}$ \\ ${ }^{1}$ V.I. Vernadsky Crimean Federal University, Simferopol, Russia \\ ${ }^{2}$ V.I. Vernadsky Crimean Federal University, Simferopol, Russia
}

\begin{abstract}
The article deals with the concept of emotional intelligence as a structure-forming component of the future teacher's psychological culture. The paper substantiates the need to develop this personal quality in future teachers, as well as its importance for successful professional activity. The article analyses the structural components of emotional intelligence in the personal sphere of future teachers. The study provides empirical material for the primary cross-section of diagnostics of emotional intelligence in the personality structure of future teachers.
\end{abstract}

\section{A problem statement}

Modern professional pedagogical education is largely associated with the idea that students in the process of mastering the main professional educational program develop a high level of mobility and adaptability to the inevitably changing conditions of modern society.

The teaching profession raises high emotional tension, which is a significant psychological and pedagogical problem, both in the course of work and professional training for professional and personal growth of a teacher.

The nature of the emotional tension of pedagogical activity is manifested in the forcing of experiences, which contributes to the reduction of the teacher's ability to perform effectively and the development of specific unproductive mental reactions.

Emotional tension affects the stability of behaviour and self-control.

In this state, the teacher most often demonstrates irritability, impatience, which is explained by "external" reasons.

\subsection{The objective of the work}

When writing the article, we used methods of analysis of scientific literature, periodical articles and publications on the topic, as well as empirical methods of data collection such as tests and questionnairesThe title is set in bold 16-point Arial, justified. The first letter of

\footnotetext{
${ }^{*}$ Corresponding author: ipcs-profped@yandex.ru
} 
the title should be capitalised with the rest in lower case. You should leave $22 \mathrm{~mm}$ of space above the title and $6 \mathrm{~mm}$ after the title.

\section{Materials and the results of the research}

Improving emotional stability and, as a result, professional skills of the teacher is possible through the development of emotional intelligence. According to I.N. Andreeva, emotional intelligence is "emotional literacy", "emotional competence" of a modern teacher [1]. Emotional intelligence is a psychological component of a person, which contains such components as: the ability to distinguish, understand and manage both other people's and their own emotions, as well as emotional states. Emotional intelligence acts as a systemforming factor of productivity of pedagogical activity, mediating the acquisition of professional knowledge and skills, and contributing to the personal development of the teacher and their students $[1,2]$.

The word "emotion" comes from the Latin "motere", which means "to move", so emotions are the motivating forces of the psyche in different areas of life. Emotional intelligence is understood as the emotional and intellectual activity of a person, which includes a set of personal emotional and social abilities that form the skills: to overcome the pressure of the environment; to function based on their own feelings, desires, and needs; to recognize represented emotions; to manage the emotional sphere based on the congruence of mental operations and the emotional and sensory sphere [2].

Based on the fact that the professional activity of a teacher is predetermined by a high level of responsibility and tension, the ability to emotionally-volitional regulation, the presence of dynamic interpersonal interactions, so in our opinion, the phenomenology of emotional intelligence is most effective in the process of training future teachers.

Today, it is customary to distinguish three most important auxiliary skills that form the emotional potential of a future teacher: the ability to actively listen, understand nonverbal communication, and adapt to a wide range of emotions [2]. So, considering the structure of emotional intelligence, we can distinguish the following components: empathy, emotional restraint and stability, balance, and communicative tolerance. We will reveal the content of these structural components in pedagogical activity.

Self-consciousness. Teachers with a high level of emotional self-awareness track their internal, dynamic feelings, evaluate them and understand their impact on their own feelings and psychological states, as well as on students [3]. Based on their own values, they are able to intuitively choose an effective and more acceptable way of behavioural response in various situations. A high level of emotional self-awareness makes it possible to perceive a complete picture of what is happening, which allows you to give a fair and sincere assessment, to be open in your own feelings and ideas. Teachers with a developed emotional self-awareness are more likely to show willingness to learn new skills, accept constructive criticism of their work, can ask for help, and do not hide their difficulties. Knowing your own potential and capabilities allows you to effectively use your strengths without losing your sense of reality.

Self-checking. A teacher who has learned to manage their emotions is able to handle a whole range of different feelings. If the teacher controls this process, they will be able to use emotions to achieve their goals and motivate themselves. D. Goleman suggests considering self-control through the following components: control of emotions, sincere expression of one's feelings, ability to adapt, will to win, initiative, and optimism $[2,3]$. Teachers with a high level of self-control are able to recognize and control negative, destructive emotional impulses and, if necessary, use them in a positive way. A high level of self-control allows you to manage your feelings even in conditions of severe distress, and it, in its turn, leads to calmness and reasonableness [4]. 
Openness. A teacher, who finds the opportunity to be honest with themselves and others, is in harmony with their values. The ability to recognize mistakes and failures, and the sincere expression of your feelings and beliefs contribute to professional relationships.

Adaptability. The ability to quickly adapt to a situation is one of the most important qualities of a teacher. If the teacher is adaptable, they are able to accept diverse requirements; they are active in a stressful situation, and feel comfortable in constantly changing conditions. Adaptability provides first of all flexibility of thinking and optimism $[5,6]$.

Purposefulness. Teachers with a developed will to win are guided by high personal and social standards that contribute to improving professional and personal qualities, the quality of tasks performed and the effectiveness of students' activities, achieving "acme" [6]. A teacher with the potential for purposefulness sets goals for themselves and students that require some costs, since they are able to calculate risks.

Initiative. A high rate of initiative expresses the desire for independent endeavours; there are ideas that are embodied in the educational process of educational institutions. With enthusiasm and healthy drive, fresh, innovative ideas and solutions open up new opportunities for developing educational and professional potential [2, 6].

Optimism. The teacher is an optimist who will see an opportunity in various situations. This approach to positive perception of situations, people, etc. Is predictive and effective for all subjects of the educational process. The main postulates of humane pedagogical thinking are belief in the child's capabilities; disclosure of the original nature in the child; respect and approval of their personality; directing him to the service of good and justice [7].

Pedagogical empathy, i.e. the teacher's understanding of mental states, emotions, feelings, and experiences of students, is associated with compassion. Relationships between people show emotional and personal openness, a psychological attitude to take into account each other's current states, and a certain level of trust. Teachers who have the ability to empathize are able to interact with other people's experiences, tune in to a wide range of emotional signals. [7, 8]. This personal quality allows you to build productive relationships, navigate and adapt in society, regardless of situational patterns.

One of the components of emotional intelligence is relationship management. It can be implemented through the ability to engage others, influence them, and promote positive changes. This substructure also includes the ability to resolve conflicts $[9,10]$.

Ability to engage others. Teachers who have these qualities set goals that go beyond the general tasks, set an example that can inspire and inspire.

Influence. For a professional teacher ability to influence students is very important and techniques to do it are diverse: voice inflection, tone, rhythm of presentation (depends on the listener), proof, persuasion, the ability to attract, interest, etc [11].

Help in self-improvement. A teacher who is interested in the results of their activities will strive to develop the potential abilities of students, show interest in their personality, ideals and beliefs. Help in self-improvement consists in the desire to see their goals, advantages, help to circumvent shortcomings, guide and support. Such mentors are able to give valuable advice to their wards in a timely manner $[9,11]$.

Conflict resolution. Teachers who are able to avoid conflict situations, resolve differences, withstand direct confrontation and openly analyse the claims of all parties can understand and determine the point of contact that everyone can share. The ability to bring the conflict to the surface, to accept the feelings and positions of the opponent allows you to direct energy towards a common solution $[12,13]$.

In order to study emotional intelligence as a structure-forming component of the psychological culture of the future teacher, we conducted a cross-section, which was attended by 48 students of the V.I. Vernadsky Crimean Federal University. Methods of 
empirical research: "Method of determining character accentuations" by LeonhardShmishik, the method "Motivation of success and failure" by A.A. Rean, diagnostics of "Emotional intelligence" by N. Hall, the questionnaire on emotional intelligence by D.V. Lucine $[6,14,15]$.

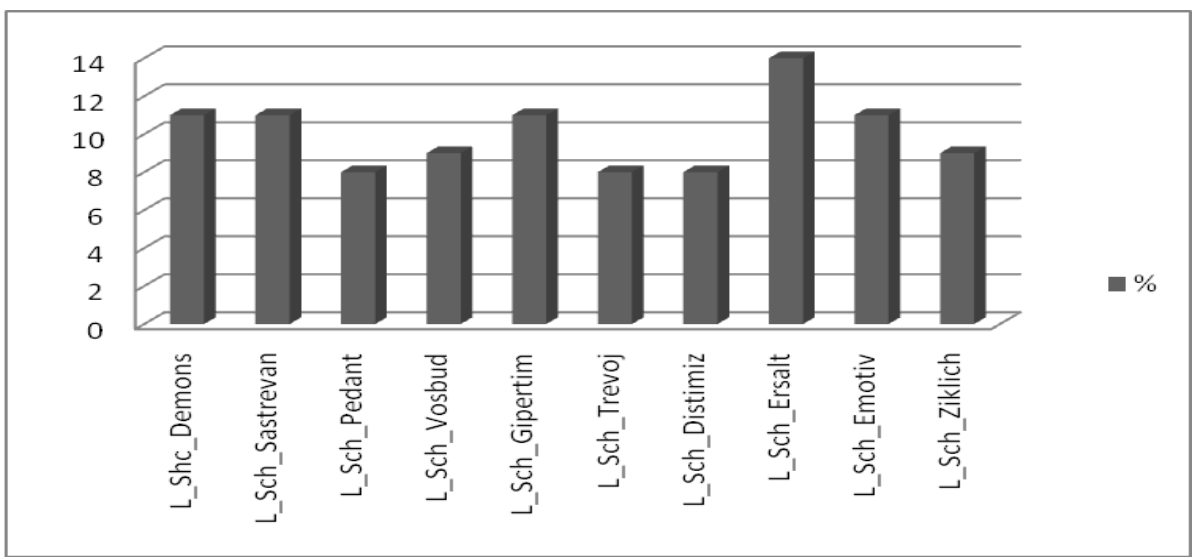

Fig. 1. Histogram of percentages of indicators of character accentuations in 48 people, the method "Method for determining character accentuations" by Leonhard-Shmishik.

The results obtained using the method "Method for determining character accentuations" by Leonhard-Schmishik indicate that in general, the predominant type of character accentuations in the considered group of students is the affective-exalted type, the accentuation is "introetive", which indicates that a large range of emotional states prevails in this sample, from delight or joy to complete despair or sadness. A bright personality trait of the majority of respondents is the ability to admire, as well as to smile, a sense of happiness, joy, and enjoyment. According to the interpretation of the method, students are characterized by high contact, talkativeness, amorous affairs; such people often argue, but do not bring matters to open conflicts, they are subject to momentary moods, impetuous, easily move from a state of delight to a state of sadness. Lability of the psyche is peculiar for them. In conflict situations, they can be both active and passive parties. They are close to friends and family, altruistic, have a sense of compassion, good taste, show brightness and sincerity of feelings.

From the data obtained, according to the method "Motivation of success and failure" by A.A. Rean, it is clear that the most pronounced in the subjects is the trend aimed at motivating success $63 \%$. According to A.A. Rean it should be assumed that the motivational pole is not clearly expressed. A high level of motivation for success in $34 \%$ means positive motivation, in which the student, starting a business, has in mind the achievement of something constructive, positive. Students' activity is based on the hope for success and the need to achieve success. They are usually confident, self-confident, responsible and active. They are distinguished by perseverance in achieving the goal, purposefulness.

And only 3\% showed a motivation aimed at failure, which refers to negative motivation. With this type of motivation, a person's activity is associated with the need to avoid failure, censure, punishment, or failure. In general, this motivation is based on the idea of avoiding negative expectations. Starting a business, the student is already afraid of possible failure in advance, thinking about ways to avoid this hypothetical failure, and not about ways to achieve success. A student who is motivated to fail is usually characterized 
by increased anxiety, low self-confidence, avoid responsible tasks, and if it is necessary to solve over-responsible tasks can fall into a state close to panic.

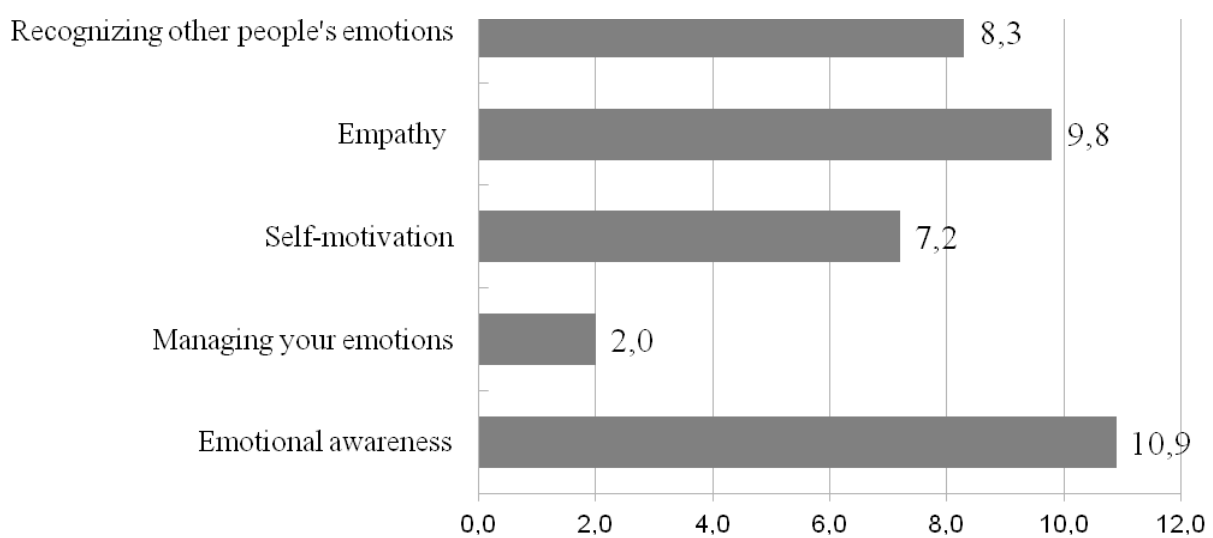

Fig. 2. Histogram of percentages of emotional intelligence indicators in 48 people, method "Diagnostics of emotional intelligence" by N. Hall.

Analysis of the results using the method "Diagnostics of emotional intelligence" by $\mathrm{N}$. Hall, shows that on average, students have a high level of emotional awareness and empathy, while a low level of control over their own emotions. Thus, we can talk about a high level of perception of emotional patterns, both our own and those around us, in the absence of self-regulation skills in various situations.

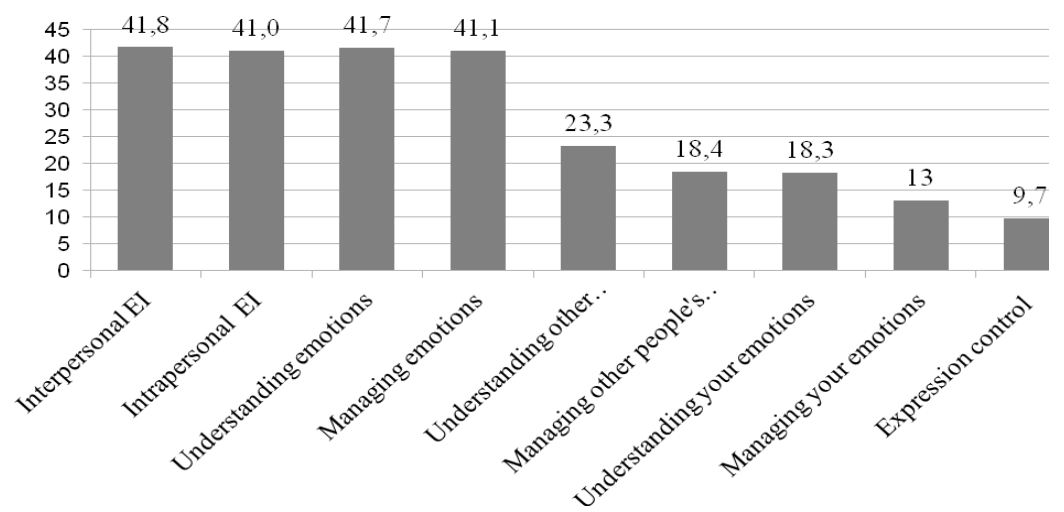

Fig. 3. Histogram of percentages of emotional intelligence indicators in 48 people, the method "Questionnaire on emotional intelligence" by D.V. Lucine.

According to the results of the "Emotional intelligence questionnaire" method by D.V. Lyusin [14], the majority of respondents have a high level of interpersonal and intrapersonal emotional intelligence, with a low level of control over expression and management of their own emotions. 


\section{Conclusions}

Thus, having considered the content and structure of emotional intelligence as a structureforming component of the future teacher's psychological culture, we can state its significance in the system of professional training. Research has shown that a high level of emotional intelligence ensures success, both in the social and personal spheres of life. A low level of emotional intelligence leads to difficulties in understanding and defining one's own emotions, and as research has shown, in controlling and self-regulating the emotional sphere, which increases the risk of psychosomatic diseases and professional burnout in future teachers. It follows that the formation of skills to understand and manage your own feelings is a personal factor that strengthens psychological and somatic health.

\section{References}

1. I.N. Andreeva, Gender differences in the structure of emotional intelligence, 282-285 (2004)

2. I.N. Andreeva, Prerequisites for the Development of Emotional Intelligence, 16-26 (2007)

3. I.N. Mescheryakova, Formation of emotional intelligence of psychology students in the process of studying at a university, 157-161 (2010)

4. R. Bar-On, The Bar-On model of emotional-social intelligence (ESI) 13-25 (2006)

5. M.J. Sanchez-Ruiz, R. Merhi, M. Nicolaou, The role of trait emotional intelligence in appearance perception $932-944$ (2020)

6. D.V. Lyusin, Modern ideas about emotional intelligence 29-36 (2004)

7. O.A. Rudakova, Emotional intelligence in the personality structure of a future Psychologist 127-130 (2020)

8. M. Vaquero-Dieg, P. Torrijos-Fincias, M.J. Rodriguez-Conde, Relation between perceived emotional intelligence and social factors in the educational context of Brazilian adolescents 33, 256-260 (2020)

9. E.Yu. Ponomareva, Essential Characteristic Of The Nature Of Emotional Intelligence 47, 102-106 (2019)

10. E. Yu. Ponomareva, Psychological and Pedagogical Aspects of Emotional Competence of Future Psychologists, 65, 341-344 (2019)

11. O.A. Rudakova, Psychological Content of Professional Training of Future Teachers, 130-145 (2018)

12. O.I. Vaganova, I.N. Odarich, O.A. Rudakova, Level differentiation technology in vocational Education 42-49 (2020)

13. M. Vaquero-Dieg, P. Torrijos-Fincias, M.J. Rodriguez-Conde, Psicologia-reflexao e critica 33 November (2020)

14. P. Lowicki, M. Zajenkowski, D. Linden, The Interplay Between Cognitive Intelligence, Ability EmotionalIntelligence, and Religiosity 59, 2556-2576 (2020)

15. D.V. Lyusin, Questionnaire for emotional intelligence Emin: new psychometric information 264-278 (2009) 